\title{
The relationships between judged similarity, judged association, and normative association
}

\author{
BERTRAM E. GARSKOF, MICHIGAN STATE UNIVERSITY \\ WILLIAM FORRESTER, RUTGERS, THE STATE UNIVERSITY
}

Word pairs varying in word association probability between members of the pairs were used to obtain judgements of similarity (JS) and three different sets of association strength judgements. Correlations between JS and associative juidgewere low with the highest reaching. 33 . Correlations between IS and normative association strength were also low. Some correlations between associative judgements and normative values were high with the correlation between bi-directional associative judgements and bi-directional norms reaching 98 .

The impetus for the research stemmed from a study by Haagen (1949) in which a .90 correlation was found between judged association strength (JA) and judged similarity (JS) for a set of synonym pairs. All synonym pairs, even those rated lowest in JS possess a relatively high degree of similarity in meaning while normative word association data typically show that synonyms are not usually strong associates. Thus, the pairs used by Haagen were restricted to the high portion of the similarity range and to the lower end of the range of normative associative strength.

It seemed worthwhile to employ Haagen's judgement instructions with a set of pairs containing both a greater variety of pair types and a wider range of normative association strength than was present in the Haagen study to increase our information concerning this issue.

Another aim of the study was to investigate the relation between association judgements and normative probability. Haagen's association instructions did not identify clearly the direction of the association to be judged. His Ss were asked to judge the degree to which each word called the other to mind and were left free to consider either word as the stimulus or to estimate bi-directional association strength. In the present study two additional types of association judgements were used. These asked Ss to estimate directly uni-directional and bi-directional associative probabilities. Since normative uni- and bi-directional probabilities were available for the pairs used, the relation between each type of association judgement and normative probability was determined.

Method

Word pairs. Fifty-one pairs were selected from a large set of word association norms collected by the senior author at Douglass College, Rutgers University. The $\mathrm{N}$ for these norms ranged between 150 and $200 \mathrm{Ss}$. The pairs were selected to sample a wide range of normative uni-directional associative probability $(.11-.88)$. Each pair consisted of a stimulus word and its most frequent associate. No opposite pairs were included. Pairs were chosen to minimize apparent interpair similarity and association.

Instructions. Four sets of instructions were employed.

1. Similarity judgements (JS) were styled after those adopted by Haagen (1949). Each pair was judged in terms of the degree of similarity in meaning between the words which formed the pair. A 7-point scale was used such that 7 represented extremely high similarity and 1 denoted words which were unrelated in meaning.

2. Association instructions (JA) were styled after those adopted by Haagen (1949). The same 7-point scale was used with 7 representing extremely high association (an association that is immediate, compelling, and consistent) and 1 used for extremely low association (one member of a pair seldom or never calling the other to mind).

3. Normative associative probability judgements (NAJ). Under NAJ Ss were instructed to rate each pair in terms of "the degree to which the first word called the second word to mind." They were asked to imagine a group of 100 students and to try to estimate how many students would say the second word as their first association to the first word.

4. Normative bi-directional associative probability judgements (NBAJ). Here instructions were identical to NAJ except that Ss were asked to estimate how many people out of 100 would say the second word as their first associate to the first word and how many would say the first word as their first association to the second word of the pair. Ss were instructed to sum these two estimates in making their judgements.

All instructions included statements that Ss were to look over the first several pairs to get a feel for the list before they began, that they were to use their first reaction to each pair and that they were to consider each possible scale value before making each judgement.

Materials. Booklets were prepared which contained the 51 to-be-judged pairs typed in lower case letters with a hyphen between the members and a space for $\mathrm{S}$ judgements to the right of each pair. The word which had served as the free associational stimulus was printed at the left and the response word on the right. Four sets of booklets were prepared, each listing the pairs in a different random order.

Each set of instructions was duplicated on single sheets of paper.

Procedure. Word-pair booklets and instructions were distributed during regular meetings of introductory 
Table 1. Correlations between Similarity Judgements, Association Judgements and Normative Associative Probability.

\begin{tabular}{|c|c|c|c|c|c|c|}
\hline & JA & JS & NAJ & NBAJ & NAP & NBAP \\
\hline JA & & .33 & $\begin{array}{r}80 \\
01\end{array}$ & $\begin{array}{r}.84 \\
02\end{array}$ & $\begin{array}{r}.49 \\
25\end{array}$ & $\begin{array}{r}.84 \\
10\end{array}$ \\
\hline NAJ & & & & .83 & .54 & .77 \\
\hline NBAJ & & & & & .45 & .98 \\
\hline NAP & & & & & & .88 \\
\hline
\end{tabular}

psychology discussion sessions such that approximately equal numbers of Ss in each class received each order of words and each type of instructions. In all, 90 Ss served in the experiment. Due to inadequacies in the distribution procedure, Ns for the different instruction types were not identical $(J S=20, J A=26, N A J=21$, NBAJ $=23$ ) .

\section{Resulis and Discussion}

The data consist of six sets of values: four sets of judgements, normative uni-directional probabilities (NAP), and normative bi-directional probabilities (NBAP). Mean judgements for each pair were computed for each judgement group. The NAP was determined for each pair by dividing the frequency of $R$ to $\mathrm{S}$ by $\mathrm{N}$, the number of Ss used in the normative association procedure. NBAP values were determined for each pair by summing the frequency of $R$ to $S$ and $S$ to $R$ and dividing by $\mathrm{N}_{R-S}+\mathrm{N}_{\mathrm{S}-\mathrm{R}}$. The NAP and NBAP were normalized by means of arc-sign transformations. Pearson-product correlations were then computed between each set of values. Table 1 presents the results of these computations.

Examination of these correlations indicates that JS did not correlate highly with any measure of association. The highest correlation found, that between JA and $J S$, although significant $(p<.05)$, accounted for less than $10 \%$ of the variance. These correlations suggest that for the pairs used association strength and similarity in meaning are distinct types of word relatedness which do not covary.

Previous unpublished data attest to the reliability of the present finding. The present senior author and Houston (1963) collected JA and JS judgements for each stimulus word in the Jenkins-Russell norms (1954), paired with its primary associate. The procedure adopted was identical to that used in the present study. JA, JAP, and JS judgements were collected for the 100 word pairs. Approximately $30 \mathrm{Ss}$ served in each group. The list contained many pairs of opposites which would tend to be judged low in JS but which possessed the highest NAP in the list. These pairs depressed the correlation unduly because of this built-in negative relationship. Therefore, they were omitted from the analysis and the correlations for the remaining 69 pairs were computed. The correlation between JA and JS for the remaining pairs was .32 and the correlation between JAP and JS was -.03. Thus, we have two sets of strikingly similar results. Each indicates that JS does not correlate highly with association strength. The apparently reliable finding that JA and JS are not highly correlated raises a reasonable question.

Is Haagen's original finding replicable with other sets of synonym pairs or was it, for some reason, spurious? One piece of evidence suggests the former conclusion. The 51 word pairs used in the present study were divided at the mean JS value into two groups and correlations were computed between JS and $\mathrm{JA}$ for each group. A correlation of $-.51(\mathrm{~N}=28)$ was found between JA and JS for the pairs low in JS while a correlation of $.68(\mathrm{~N}=23)$ obtained between JA and JS for the remaining pairs. These correlations, taken with the correlation reported by Haagen, strongly suggest that the correlation between JA and JS will vary directly with the level of JS. Since Haagen used pairs from the high range of JS, he found a high correlation between JA and JS.

Examination of the remaining correlations reveals that $\mathrm{HA}$ instructions led to judgements which coincided more closely to bi-directional norms than to unidirectional norms. However, NAJ also correlated more highly with NBAP than NAP. Apparently, backward association strength influenced the judgements in each procedure. Finally, it is worth noting that NBAJ and NBAP correlated almost perfectly. The NBAJ procedure may be an economical and precise method for the assessment of association strength.

\section{References}

Garskof, B. E., \& Houston, J. P. A reanalysis of the meaning similarity-associative strength relationship. Mimeo, 1963.

Haagen, C. H. Synonymity, vividness, familiarity and association value ratings of 400 pairs of common adjectives. J. Psychol., $1949,27,453-463$.

Russell, W. A., \& Jenkins, J. J. Language in behavior: The complete Minnesota norms for responses to 100 Words from the KentRosanoff Word Association Test. Tech. Rep. No. 11, N 8onr, 66216 , University of Minnesota, 1954. 